\title{
Electrostatic Energy Harvesting Systems: A Better Understanding of Their Sustainability
}

\author{
Rita T. Aljadiri, Luay Y. Taha, and Paul Ivey
}

\begin{abstract}
This paper presents a review of electrostatic energy harvesting systems. It includes several types of electrostatic harvesters. The review is done based on two main categories, capacitor structure and conversion mechanism. Since electrostatic harvesters are important devices when sustainability is considered, this review gives an in depth evaluation and comparison of all possible configurations. It should be noted that this review is based on recent published literature.
\end{abstract}

Index Terms - Conversion mechanism, electrostatic, energy harvester, variable capacitor.

\section{INTRODUCTION}

Electrostatic converters are capacitive devices in which energy conversion takes place as the plates of a variable capacitor separate or the area of the plates is modified in response to externally applied mechanical energy [1]. The main unit of an electrostatic energy harvester is its variable capacitor, which makes use of a change in capacitance to either cause a voltage increase or a charge increase in the harvesting system. Many types of harvester have been reported in previous studies based on various capacitor structures and energy transfer strategies. A literature review of those types is presented in this paper divided into four sections: work concentrating on variable capacitor structures; previous work on conversion mechanisms; a comparison of conversion mechanisms; and a comparison of various electrostatic devices.

\section{ELECTROSTATIC ENERGY HARVESTERS}

An electrostatic energy harvester consists of a variable capacitor and an energy transfer circuit as shown in Fig. 1 [1].

The electrostatic effect takes place between the parallel plates of the capacitor on which electrical charge is stored. The harvesting of electrical energy is achieved by fixing one of the plates and moving the other by an external mechanical motion to change one of the variable capacitor parameters: either plate area or plate separation [2]. This allows mechanical movement to be converted into electrical energy according to the principle of electrostatics. The details of electrostatic energy conversion principle are addressed [3].

Manuscript received January 30, 2016; revised November 16, 2016.

Rita T. Aljadiri is with Emirates Aviation University, Dubai, United Arab Emirates (Tel.: 00971508079636; e-mail: s405407@emirates.com)

Luay Y. Taha is with Windsor University, Ontario, Canada (e-mail: taha1@uwindsor.ca).

Paul Ivey is with Birmingham City University, Birmingham, United Kingdom (e-mail: paul.ivey@bcu.ac.uk).

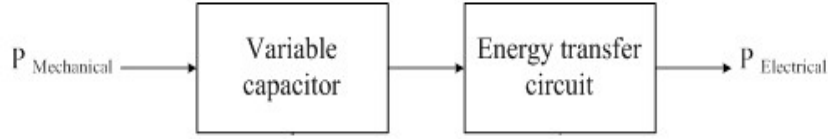

Fig. 1. Block diagram of electrostatic harvester.

\section{VARIABLE CAPACITORS FOR ENERGY HARVESTING}

The first variable capacitor for electrostatic power conversion was proposed in 1998 by Meninger and co-workers [4]. The concept was later demonstrated by Roundy and co-workers utilising large machined variable capacitors [5]. Since then, several variable capacitor structures for electrostatic harvesting have been reported in the literature. The structures of these capacitors depend mainly on changing capacitance parameters. There are three types of variable capacitor: variable area, variable gap and variable dielectric constant. These three types are considered next.

\section{A. Variable Area Capacitors}

In 1977 Sanborn F. Philp [6] reported a variable area vacuum-insulated varying-capacitance machine. This capacitor operated by rotational motion. Having evaluated its generation of high voltage direct current power, the author suggested that his capacitor machine offers two potential advantages over conventional rotating machines: first, it is capable of very high efficiency power generation; second, it is suitable for high voltage operation.

Sterken et al. [7] proposed a variable area capacitor in which the vibrations cause the capacitor plates to move in opposite directions, changing the capacitance and hence producing electricity. The author reported that experimentally $1 \mu \mathrm{W}$ could be harvested from a $5 \mu \mathrm{m}$ vibration at resonance frequency of $980 \mathrm{~Hz}$.

Tsutsumino et al. [8] developed an in-plane overlap variable area power generator. They proposed an electrode arrangement for reducing the in-plane unidirectional damping force. They confirmed that this generator model can imitate the response of the in-plane electret generator with sufficient accuracy.

Tvedt et al. [9] modelled and simulated an electrostatic in-plane overlap varying energy harvester using a circuit simulator. They investigated both linear and nonlinear models. The nonlinear model included mechanical stoppers at the displacement extremes. They used both narrow- and wide-band large amplitude excitation signals to simulate environmental vibrations. The authors found that nonlinear behaviour is significant at large displacements due to the impact on mechanical stoppers. They also suggested that for sinusoidal excitation the mechanical stoppers could cause 
output power to decrease.

Kiziroglou et al. [10] presented a novel electrostatic harvester design employing an external free-rolling proof mass. The vibration energy causes a change to the capacitor area. The authors described the fabrication process for a prototype device and the physical characterisations for dielectric sizes down to $100 \mathrm{~nm}$. They demonstrated a voltage gain of 2.4, and suggested that the device is suitable for energy harvesting from low frequency motion sources, such as the human body.

Halvorsen et al. [11] designed, fabricated and characterized a MEMS electrostatic energy harvester using a variable area capacitor. The device operates in continuous mode and features high voltage output and large travelling distance of a big mass within a compact design using full bulk silicon thickness. The output power is about $1 \mu \mathrm{W}$ at an acceleration power spectral density of $0.03 \mathrm{~g}^{2} / \mathrm{Hz}$.

O'Donnell et al. [12] investigated the feasibility for directly generating HVDC power using a novel generator topology as an alternative solution to large offshore wind farms. They used variable-capacitance based on the work of Sanborn F. Philip (1977). The authors presented a generator that uses electrostatic fields to generate HVDC output with a minimum of power conditioning, and reported that its system power density is comparable to conventional generator-transformer-rectifier systems.

Reznikov et al. [13] (2010) reported the generation of the electric power by a variable area permanently polarized capacitor used for energy harvesting from oscillations and rotation. Their experimental results demonstrated the generation of either high voltage pulses, or low voltage alternating current that need to rectified, regulated and stored. They based their design for an electrostatic swing energy harvester on the linear displacement of a conductive shield between the electrets and passive electrode. The authors provided experimental data showing the harvester could provide a DC voltage greater than $3 \mathrm{~V}$.

\section{B. Variable Gap Capacitors}

Mizuno et al. [14] was the first team to propose a variable gap method in which mechanical vibration is used to vary the gap and hence the resonating capacitance, which can then be tuned to obtain optimum output power.

Maio et al. [15] described the fabrication and initial testing of a micro-machined variable capacitor for power generation. The measured capacitance of the device varies from $100 \mathrm{pF}$ to around $1 \mathrm{pF}$. The authors presented the initial tests of the capacitor on a vibration system generating a high voltage output of $2.3 \mathrm{kV}$ when the capacitor was charged by a voltage source of $26 \mathrm{~V}$. This corresponds to an energy conversion rate of $2.4 \mu \mathrm{J}$ per cycle, or $24 \mu \mathrm{W}$ at a vibration frequency of $10 \mathrm{~Hz}$.

Despesse et al. [16] presented an in-plane gap closing structure with an interesting rectangular structure where the capacitance density can be increased by using triangles fingers. The gaps can be adjusted easily by adjusting the finger overlaps and then adapting the structure to vibration conditions in order to maximize capacitance variation. The achieved output power was $16 \mu \mathrm{W}$ per gram over a frequency band between $20-100 \mathrm{~Hz}$, with an efficiency of $60 \%$.
Pasquale et al. [17] investigated the performance of micro systems based on electrostatic coupling, and tested the power scavenged by a capacitive micro-scavenger with an out-of-plane gap-closing layout. The authors investigated the performance of this energy harvesting strategy by comparing it both to a configuration with an in-plane motion of electrostatic benders and to the performance of bimorph piezoelectric transducers.

Guillemet et al. [18] studied the vibration energy harvesting capabilities of an out-of-plane gap-closing converter. Their goal was to maximize the output energy harvested from a MEMS device while preventing the integrity of the conditioning circuit. The originality of their work lies in the optimisation, taking into accounts both electrical and mechanical aspects and studying at the same time the consequence of the voltage limitations on the transducer.

Guillemet et al. [19] presented the design and testing of an electrostatic energy harvester based on a gap-closing comb drive structure. The capacitor is made from a thick silicon wafer, whose total capacitance varies from 40 to $290 \mathrm{pF}$. The authors' results show a parasitic capacitance of $30 \mathrm{pF}$. The device was tested with a charge pump circuit for $500 \mathrm{sec}$. The harvested power was $938 \mathrm{nW}$.

Janicek et al. [20] reported a generator design based on an electrostatic converter that uses the principle of periodical modification of the gap between the electrodes of a capacitor. They designed and modelled the structure as a 3D silicon-based MEMS. The authors took an innovative approach to testing the structure under a very low resonant frequency of around $100 \mathrm{~Hz}$. They also presented the design of a long cantilever spring with a minimum area of the chip, its ability to work in $3 \mathrm{D}$ mode, and the capacity to be tuned to reach the desired parameters.

\section{Variable Dielectric Constant Capacitors}

The methodology of producing electricity by changing the dielectric materials of a capacitor using mechanical energy topology was not previously common. However, it was adopted using flow energy topology by Boland et al. in 2005 [21], who described a device that is a fixed-charged, capacitor with air-filled gaps and liquid droplets that travel by vibration. As the liquid moves in and out of the gaps, a voltage is generated across the capacitor according to the position of the droplets. The authors also studied the possibility of using serial and parallel arrays of the device to increase power output. The power output produced from the parallel arrays was around $10 \mu \mathrm{W}$.

Salem et al. [22] in 2007 presented a novel technique employing a switchable dielectric constant for enhancing the power delivered by electrostatic converters. Their approach provides power 103 times greater than that provided by conventional MEMS devices. They also considered potential technical challenges associated with viscous damping and time response of the device. They estimated the time response of the device as varying from $100 \mu$ s to $300 \mu$ s, depending on the device size.

Talal [23] in her PhD thesis presented an electrostatic energy conversion method utilizes a change in the dielectric constant of the material between capacitor plates. The author suggested that by changing the actual material between the 
plates, it pumps energy into a storage device. The devices have fixed capacitance geometries such as gap and area but modify the dielectric permittivity by allowing a set of two fluids with two different dielectric constants to flow between fixed plates.

\section{Electrostatic Harvester CONVERSion MeChanisms}

There are three main techniques used for the electrostatic conversion mechanism: switched constant charge systems, switched constant voltage systems and continuous electret-based systems.

The electret-free capacitors operate in either charge- or voltage-constrained systems so there are two possible extremes of operation, as shown in Fig. 2. In both cases, the mechanical work done is converted into electrical energy. In this method harvesters need an external power source for pre-charging the capacitor in order to generate electricity.

On the other hand, the electret-based devices are very similar to electret free devices. The main difference is the additional electret layers that are applied to one or both plates of the capacitor for polarisation purposes. Currently available electrostatic harvesters tend to use electret-based devices more. However, the manufacturing process is complicated, being very similar to that for magnets. It entails injecting an excess of charge in a dielectric layer or heating the dielectric layer above its melting temperature. The layer is then left to cool down to maintain an electric field. This allows the dipoles of the dielectric layer to be oriented in the same direction as the electric field [1], as shown in Fig. 3.

A summary of the various conversion mechanism is presented next based on literature published in the last few years.

\section{A. Switched Constant Voltage Systems}

Torres et al. [24] in 2009 reported a voltage-constrained electrostatic CMOS harvester. The authors explained how much energy is available in such a system before and after harvesting, and offered energy conversion schemes for increasing net energy gain during all operational phases.

In the same year, the same group of researchers [25] reported a prototype circuit that pre-charges, detects and synchronizes to a variable voltage-constrained capacitor. The authors verified experimentally that it is possible to harvest energy using the electrostatic principle. Their experimental results showed that the system harvests $9.7 \mathrm{~nJ} /$ cycle by investing $1.7 \mathrm{~nJ} /$ cycle, for a net energy gain of approximately $8 \mathrm{~nJ} /$ cycle at an average of $1.6 \mu \mathrm{W}$ for every $200 \mathrm{pF}$ variation.

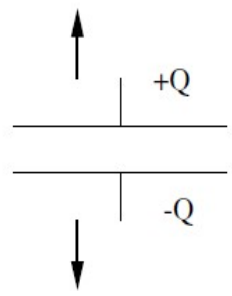

(a)

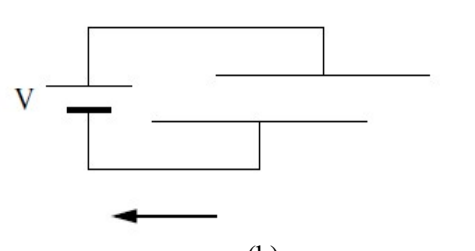

(b)

Fig. 2. Two methods of obtaining energy using a variable capacitor: (a) Constant charge; (b) Constant voltage.

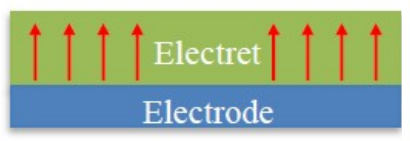

(a)
Electret

Electrode
Fig. 3. Electret based electrostatic converters. (a) Dipole orientation and (b) Charge injection.

Torres et al. [26] in 2010 reported a self-tuning battery-constrained electrostatic energy harvester integrated circuit IC that adapts to changing battery voltages to produce usable power from vibrations across the entire battery voltage operating range. Their prototype keeps the variable capacitor voltage constant so that kinetic energy in vibrations can generate and direct current into the battery when capacitance decreases. The reported pre-charger circuit is unlike other inductive based circuits, which charge the variable capacitor to battery voltage and adapt to a constantly shifting battery voltage. The proposed pre-charger circuit has a self-tuning reference system.

Kwon et al. [27] presented a design for low-power switched-inductor converters capable of producing high net energy gains, using a switched-inductor, voltage-constrained electrostatic power stage.

\section{B. Switched Constant Charge Systems}

Haas et al. [28] presented a novel voltage step-up converter based on a charge-constrained variable parallel-plate capacitor in combination with an electrostatic actuator. Electrical equivalent circuit and system level Simulink models were developed. Based on these models, the authors analysed the design parameters and expected device performance to provide a starting point for prototype implementation.

Yen et al. [29] presented a design for a variable capacitance vibration energy harvester that combines an asynchronous diode-based charge pump circuit with an inductive energy flyback circuit to deliver $1.8 \mu \mathrm{W}$ to a resistive load. The complete harvester operates under constant charge with only one gated MOSFET for energy flyback control, which greatly simplifies switching control and avoids time management issues. The authors explained that the system requires a start-up voltage of less than $89 \mathrm{mV}$, indicating that it can be initially charged by a piezoelectric film.

Galayko et al. [30] presented an analysis and system design for a capacitive harvester of vibration energy composed from a mechanical resonator, capacitive transducer and a conditioning circuit based on the buck DC-DC converter architecture with auto-adaptation to external vibration changes. The authors explained in detail the charge pump operation and presented a QV diagram of the operation.

Dudka et al. [31] designed and modelled a calibration block for a harvester based on a charge pump concept which adapts the operating parameter of the conditioning circuit to the external vibrations. They improved on the basic architecture by adding a second output to the existing flyback circuit DC-DC convertor. This allows low DC voltage to be generated for the load without using an additional DC-DC convertor, which would require an additional inductor. 
Raafat et al. [32] proposed a design for a piezoelectric voltage generator integrated with electrostatic structure to provide the necessary voltage. In this way, the system is self-initializing and autonomous. The harvester is CMOS-compatible based on a charge pump circuit.

Florentino et al. [33] reported a variable capacitor and switch circuit architecture for the buck converter. The buck converter is used to step down the voltage harvested when the charge applied across the capacitor is constant. The authors investigated the performance of the switch circuit in relation to the amount of energy loss, suggesting that the main reason for losses is the size of the transistor. They developed their circuit in the Cadence software using high voltage components that can support voltages up to $50 \mathrm{~V}$. The presented circuit loses less than $4 \%$ of transferred energy.

Kempitiya et al. [34] presented an electrostatic generator with asynchronous control and charge flyback mechanism to optimise the useful energy generated by the harvester. The authors' theoretical and experimental investigations showed that there is an optimum value for the storage capacitor and the cycle number for maximum scavenging of ambient energy. The analysis also indicated that the most energy can be harvested when mechanical and electrical events are synchronised. Their theoretical results were confirmed by measurements on an electrostatic converter prototype with an asynchronous energy harvesting circuit.

\section{Continuous Electret Based Systems}

Sterken et al. [35] proposed a device with a vibration sensitive variable capacitor polarized by an electret as new approach to mechanical energy harvesting using an electrostatic converter. The vibration causes changes in the capacitance resulting in harvesting current to a load circuit. The authors proposed a prototype design based on an MPW-service. The prototype was able to generate power of $50 \mathrm{pW}$.

Naruse et al. [36] developed an electrostatic micro power generator with vibration structure and new electret electrode for low frequency energy harvesting applications. The authors suggested that it has the advantage of being usable for high power structures because of both the controlled gap between electrodes and the low frequency vibrations. The generator was able to generate a $40 \mu \mathrm{W}$ of power output at very low frequency vibrations of around $2 \mathrm{~Hz}$.

Edamoto et al. [37] developed a vibration-based electret generator for energy harvesting applications. The spring material used was Parylene for low frequency vibrations. The adopted structure was a gap change for capacitance variation. At frequencies as low as $21 \mathrm{~Hz}$, a large in-plane amplitude of $0.5 \mathrm{~mm}$ was achieved. The authors demonstrated LED operation from the generator using a low-power-consumption impedance conversion circuit.

Miki et al. [38] developed an electret power generator driven by electromechanical vibration. The experimental data were directly compared with the model characteristics. The authors found that the circuit parameters should be optimized depending on the oscillation conditions. They also found that the electrostatic damping force acting on the seismic mass depends on the power management and rectification circuits.

$\mathrm{Bu}$ et al. [39] reported an electrostatic energy harvester with an inter-digitised capacitive structure biased by electrets. The work proposed a novel collision-based energy conversion method. Based on their theoretical results, they proposed a method that generates high coupling efficiency at low vibration frequency. The authors found that the device is capable of harvesting output power of $0.58 \mu \mathrm{W}$ and an available power density of $41.67 \mu \mathrm{W} / \mathrm{mm}^{3}$ at $2 \mathrm{~Hz}$ collision input. The authors suggested that the collision method could be used to harvest vibration energy at random and low frequencies.

Suzuki et al. [40] presented the concept, design, simulation, fabrication and evaluation of a novel micro generator based on electrostatic induction. The authors developed an asymmetric electret gammadion spring electrode for wide band vibration with three vibration modes. For mode 1, the maximum power output generated was $7.48 \mathrm{nW}$ at $110 \mathrm{~Hz}$; for mode 2, it was $2.19 \mathrm{nW}$ at $165 \mathrm{~Hz}$; and for mode 3 it was 1.72 $\mathrm{nW}$ at $243 \mathrm{~Hz}$. They suggested that their method solves the problem associated with narrow operation frequency bandwidth, which strictly limits power output under normal wideband vibrations.

Altena et al. [41] in 2013 presented many improvements for an electret based electrostatic harvester. The device was initially able to generate a power output of $175 \mu \mathrm{W}$ but after improvement with a higher resonance frequency and higher electret potential, it was capable of generating $497 \mu \mathrm{W}$ AC power, which is the highest reported power for electret based harvesters. The authors suggested two improvements to the design: first, improvements to the electrical connections of the harvester; second, to the electrode geometrical setup.

\section{COMPARATIVE StUdiES OF CONVERSION MECHANISMS}

A number of comparative studies of the switched constant charge and constant voltage systems have been reported in the literature. A summary of these studies are presented in this section.

Meninger [42] and a group of researchers at MIT working on electrostatic generators were the first group to present an electrostatic micro generator work in the literature. They described and compared in detail the constant charge and constant voltage approaches. By using QV charge-voltage cycle plots, they reported that constant voltage operation is better than constant charge operation for maximising power generation. The authors were capable of achieving constant voltage operation by attaching a large fixed capacitor in parallel with the variable capacitor. They noted that the larger the parallel capacitor the more power is generated, although more initial energy loss occurs.

Further information on constant charge and constant voltage is given in Miranda's Ph.D. thesis [43]. The author presented a simulation of a variable capacitor and power electronics in Matlab, using the parameters of an off-the shelf MOSFET. The author concluded that power electronics for constant charge are easier to implement than constant voltage. However, constant voltage systems generate low level voltages within the breakdown limits of standard IC process technologies.

Mitcheson et al. [44] compared three main types of 
electrostatic micro generator conversion mechanisms: constant voltage, constant charge switched and electret based continuous. The authors considered that the switched types can be controlled easily to perform maximal power point tracking. However, this increases the complexity of the associated circuitry required to control the switching devices. The authors also suggested that, for large electrostatic forces, constant charge designs are preferable.

Mitcheson and his team studied the power processing requirements of electrostatic harvesters, specifically for the constant charge approach. The authors highlighted issues with this approach, explaining that the harvester works with small amounts of charge at high voltage. The authors described how, when the generator experiences acceleration, the capacitance of the variable capacitor decreases and the voltage rises. In typical operation, the voltage generated on the capacitor plates can reach a few hundred volts so the harvested voltage must be stepped down to lower voltages to be suitable for powering low power loads. The suggested solution for this problem was the use of a fly-back step-down converter.

Beeby et al. [45] reviewed the use of kinetic energy harvesting as a potential power supply for wireless applications. The authors studied three types of electrostatic harvesters: in-plane overlap converters; in-plane gap closing converters; out-of-plane gap closing converters. The authors suggested that all the three types can be operated in both charge-constrained and voltage-constrained cycles. They argued that the voltage-constrained approach generates more energy than the charge-constrained approach. However, by using a parallel capacitor in charge-constrained systems, energy levels can reach those of voltage-constrained systems because parallel capacitors effectively constrain the voltage across the energy harvesting variable capacitor.

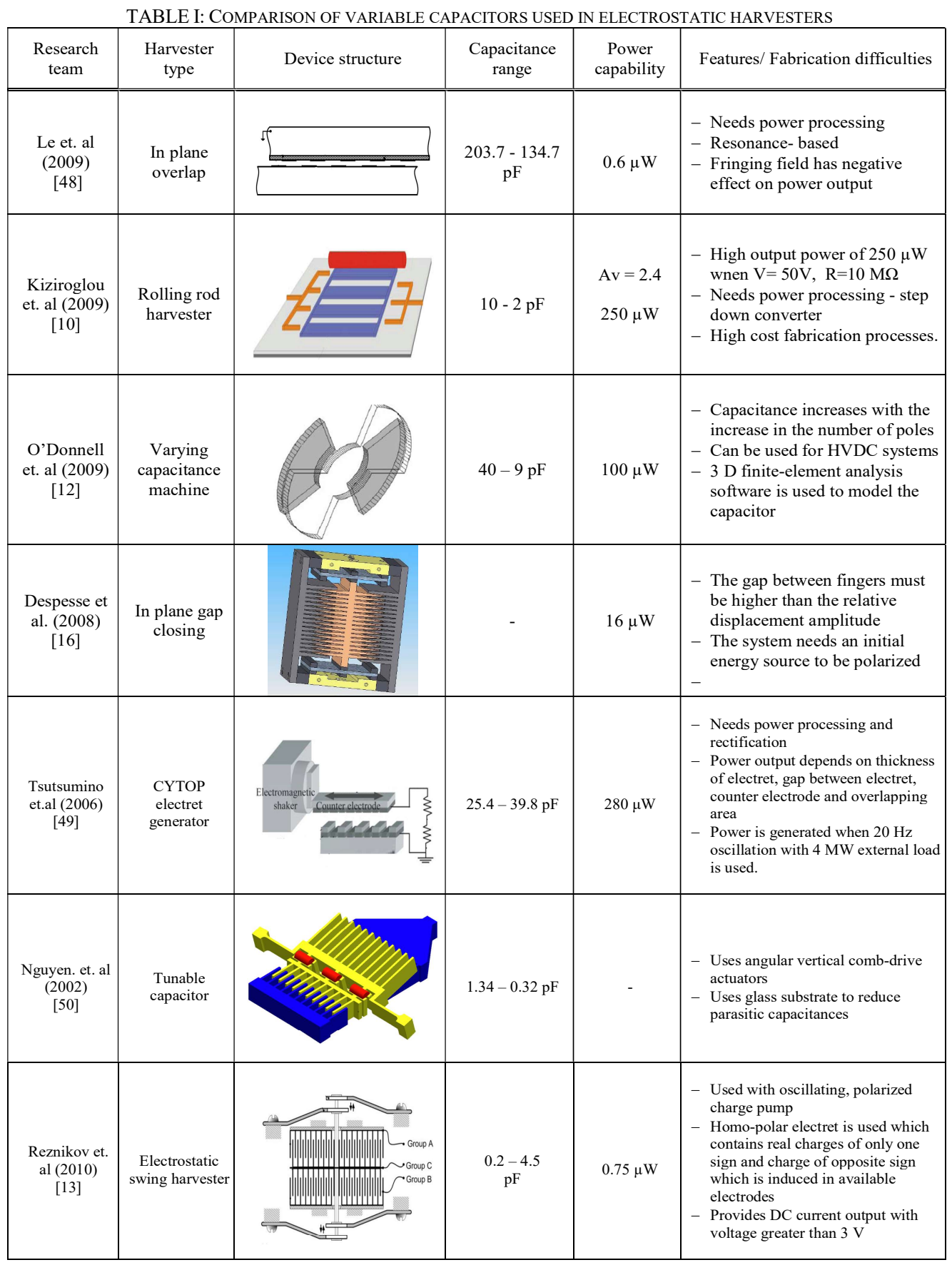




\section{Comparative Studies of CAPACITOR Structures}

Roundy et al. [46] presented a useful comparison between in-plane overlap and gap-closing converters as shown in Fig. 4. The authors suggested that the number of fingers for the in-plane gap closing converter is a function of the maximum deflection of the flexures; this is due to the fact that the fingers must be spaced far enough apart to accommodate the displacement. Therefore, lower maximum and minimum capacitances result from higher spring deflections. However, this is not the case with in-plane overlap converters, where more power is harvested when larger spring deflections are applied. The authors claim that in-plane overlap designs are more sensitive to parasitic capacitance than gap-closing converters.

Lim et al. [47] compared detailed models of three different design concepts: in-plane overlap converter; in-plane gap-closing converter; out-of-plane gap-closing converter. The authors evaluated and compared the three design concepts based on simulations and practical considerations. The simulations results indicated that the highest power density is available from in-plane gap-closing converters, followed by out-of-plane gap-closing converters and finally in-plane overlap converters. The authors suggested that, to improve the power capabilities of the in-plane overlap, the surface area of the capacitor plates needs to be increased to produce more energy.

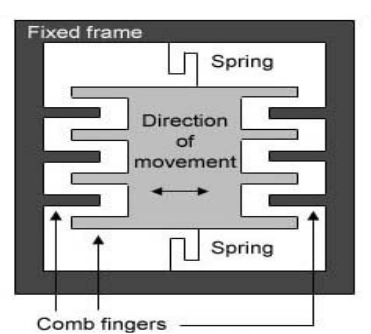

(a)

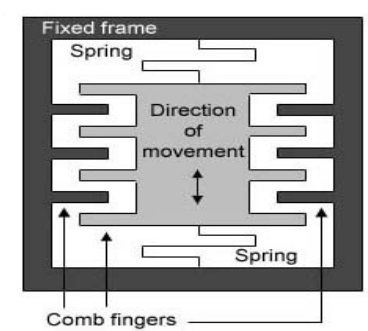

(b)

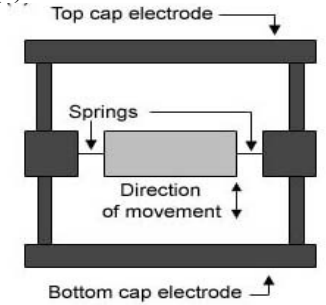

(c)

Fig. 4. Vibration-based electrostatic harvesting devices (a) In-plane overlap converter (b) In-plane gap-closing converter and (c) Out-of-plane gap-closing converter.

Boisseau and group of researchers in 2012 reported on the assessments, limits and perspectives of various types of electrostatic energy harvester. One of the main limitations was capacitor gap control. This is an important factor that affects output power as it is greatly linked to capacitance variations that must be maximized. Therefore, the authors suggested that the air gap must be controlled precisely and minimized to achieve high capacitances. Moreover, it is also important to prevent from electrical breakdown problems [1].

Six main types of variable capacitors used for energy harvesting from previous studies were compared in this paper. A summary of the results is given in Table I.

\section{CONCLUSION}

Electrostatic harvesters are compact converters with a simple structure. The energy density of these devices can be easily modified by changing the structure of the capacitor. This represents a potential solution to developing the market of energy harvesting powered applications by making it possible to develop low-cost energy harvesting devices. The design of an electrostatic energy converter depends mainly upon the structure of the variable capacitor and the conversion mechanism approach. Research studies have presented a wide range of possible structures and approaches that can be adopted. However, the proposed structures and approaches presented in the literature so far have both advantages and drawbacks.

The choice of capacitor structure depends on the type of mechanical energy applied: rotational motion or vibration. Both types can be extracted from kinetic energy. Variable area capacitor appears to be more suitable for harvesting rotational energy. Area of the capacitor plates can be modified easily by the rotational mechanical energy. While a variable gap capacitor works mostly with vibrations. Moreover, variable area structures can be easily fabricated to electret free - air capacitors. It is relatively easy to increase capacitance by increasing the surface area of the capacitor plates.

The choice of conversion mechanism depends on the variable capacitor structure. As highlighted in the paper, the conversion mechanism can use either continuous or switched systems. The continuous approach is an electret-based approach that requires a polarisation source and special fabrication processes. While the switched constant voltage and constant charge approaches are easier to implement, they require switching control circuitry. Comparing the constant voltage and constant charge approaches, it is noticeable that the former offers more energy. Therefore, it is more appropriate for electrostatic energy harvesting provided that it generates low voltages within the breakdown limits of standard IC process technologies. On the other hand, the constant charge approach fits few types of electrostatic harvesters, especially for large electrostatic forces. The electrostatic force depends on the dimensions of the capacitor, the proof mass and the operating condition. It is more difficult to achieve high electrostatic forces using constant voltage structures than constant charge structures. For this reason, constant charge is more suitable when large electrostatic forces are required.

\section{REFERENCES}

[1] S. Boisseau, G. Despesse, and Seddik, "Electrostatic conversion for vibration energy harvesting," Small-Scale Energy Harvesting, 2012.

[2] Miranda, "Electrostatic vibration-to-electric energy conversion," $\mathrm{PhD}$ thesis, Massachusetts Institute of Technology, Massachusetts, USA, 2004.

[3] R. T. Abdulmunam, L. Y. Taha, and P. Ivey, "Electrostatic harvester for wind energy harvesting and wind speed remote sensing," in Proc. IEEE Canadian Conference on Electrical and Computer Engineering, Canada, 2015.

[4] S. Meninger, J. M. Miranda, J. L. A. Chandrakasan, A. Slocum, M Schmidt, and R. Amirtharajah, "Vibration to electric energy conversion," IEEE Transactions Very Large Scale Integration (VLSI) Systems, vol. 9, pp. 64-76, 2001. 
[5] S. Roundy, "Energy scavenging for wireless sensor nodes with a focus on vibration to electricity conversion," $\mathrm{PhD}$ Thesis, University of California, Berkeley, 2003.

[6] S. F. Philp, "The vacuum-insulated, varying capacitance machine," IEEE Transactions on Electrical Insulation, vol. 12, no. 2, pp. 130-136, 1977.

[7] T. Sterken, K. Baert, G. Borghs, P. Fiorini, and R. Puers, "An electret-based electrostatic $\mu$-generator," in Proc. the 12th International Conference on Solid State Sensors, Actuators and Microsystems, pp. 1291-1294, 2003.

[8] T. Tsutsumino, Y. Suzuki, and N. Kasagi, "Electromechanical modeling of micro electret generator for energy harvesting," in Proc. The 14th International Conference on Solid State Sensors, Actuator and Micro Systems, Lyon, France, pp. 863-866, 2007.

[9] L. G. W. Tvedt, L. C. J. Blystad, and E. Halvorsen, "Simulation of an electrostatic energy harvester at large amplitude narrow and wide band vibrations," in Proc. the DTIP MEMS and MOEMS, pp. 296-301, 2008.

[10] M. E. Kiziroglou, C. He, and E. M. Yeatman, "Rolling rod electrostatic microgenerator," IEEE Transactions on Industrial Electronics, vol. 56 no. 4, pp. 1101-1108, 2009.

[11] E. Halvorsen, E. R.Westby, S. Husa, A. Vog, N. P. Østbø, V. xLeonov, T. Sterken, and T. Kvisterøy, "An electrostatic energy harvester with electret bias," in Proc. Solid-State Sensors, Actuators and Microsystems Conference, pp. 1381-1384, 2009.

[12] R. O'Donnell, N. Schofield, A. C. Smith, and J. Cullen, "Design concepts for high-voltage variable-capacitance DC generators,' Industry Applications, IEEE Transactions, vol. 45, no. 5, pp. 1778-1784, 2009.

[13] M. Reznikov. (2010). Electrostatic swing energy harvester. Proceedings of ESA Annual Meeting on Electrostatics. [Online] Available: http://www.electrostatics.org/images/ESA2010_G3_Reznikov.pdf

[14] S. Mizuno, M. Ishihara, S. Wickramanayaka, and N. Miyazaki, Electrostatic Chuck Device, JUSTIA Patent, 2003.

[15] P. Miao, A. S. Holmes, E. M. Yeatman, T. C. Green, and P. D. Mitcheson, "Micro-machined variable capacitors for power generation," Electrostatics, vol. 178, pp. 53-58, 2003.

[16] G. Despesse, T. Jager, C. Condemine, and P. D. Berger, "Mechanical vibrations energy harvesting and power management," in Proc. IEEE Sensors Conference, pp. 29-32, 2008.

[17] G. D. Pasquale, E. Brusa, and A. Somà, "Capacitive vibration energy harvesting with resonance tuning," in Proc. the Design, Test, Integration \& Packaging of MEMS/MOEMS Symposium, Torino, Italy, pp. 280-285, 2009

[18] R. Guillemet, P. D. Basset, D. Galayko, F. Marty, and T. Bourouina, "Efficient in-plane gap closing MEMS electrostatic vibration energy harvester," Power MEMS 2012, Atlanta, GA, USA, pp. 137-140, 2012

[19] R. Guillemet, P. Basset, D. Galayko, and T. Bourouina, "Combined optimization of electrical and mechanical parameters of an out-of-plane gap-closing electrostatic Vibration Energy Harvester (VEH)," in Proc. 2010 Symposium Design Test Integration and Packaging of MEMS/MOEMS (DTIP), pp. 73-78, 2010.

[20] V. Janicek and M. Husak, "3D energy harvester evaluation," Radio Engineering, vol. 22, no. 1, pp. 251-258, 2013.

[21] J. S. Boland, H. W. Lo, J. D. M. Messenger, and Y. C. Tai, "Arrayed liquid rotor electric power generator system," in Proc. the 18th IEEE International Conference on MEMS, pp. 618-621, 2005.

[22] M. Salem, D. Andra, B. Tasciuc, and M. M. Hella, "Electrostatic MEMS converters with a switchable dielectric constant for micro-scale power generation," in Proc. the IEEE Microelectronics Conference, pp. 205-208, 2007.

[23] R. Talal, "Transpiration as a mechanism for mechanical and electrical energy conversion," PhD thesis, University of Michigan, 2008.

[24] E. O. Torres, A. Gabriel, and R. Mora, "Energy budget and high gain strategies for voltage constrained electrostatic harvesters," IEEE Transactions on Power and Energy, 2009a.

[25] E. O. Torres, A. Gabriel, and R. Mora, "Electrostatic energy-harvesting and battery-charging CMOS system prototype," IEEE Transactions on Circuits and Systems: Regular Papers, vol. 56, no. 9, $2009 \mathrm{~b}$.

[26] E. O. Torres, A. Gabriel, and R. Mora, "0.7- $\mu \mathrm{m}$ BiCMOS electrostatic energy-harvesting system IC," Solid-State Circuits, IEEE Journal, vol. 45, pp. 483-496, 2010.

[27] D. Kwon, A. Gabriel, R. Mora, and E. O. Torres, "Harvesting Kinetic energy with switched-inductor DC-DC converters," Georgia Tech Analog, Power, and Energy IC Research, 2010.
[28] C. H. Haas and M. Kraft, "Modelling and analysis of a MEMS approach to DC voltage step-up conversion," Journal of Micromechanics and Micro-Engineering, pp. 114-122, 2004.

[29] C. B. Yen and J. H. Lang, 'A variable-capacitance vibration-to-electric energy harvester," IEEE Transactions on Circuits and Systems-I. Fundamental Theory and Applications, pp. 1-8, 2005.

[30] D. Galayko, P. Basset, and A. M. Paracha, "Optimization and AMS modeling for design of an electrostatic vibration energy harvester's conditioning circuit with an auto-adaptive process to the external vibration changes," in Proc. Symposium on Design, Test, Integration and Packaging of MEMS/MOEMS-DTIP, 2008.

[31] A. Dudka, D. Galayko, and P. Basset, "VHDL-AMS modeling of adaptive electrostatic harvester of vibration energy with dual-output DC-DC converter," Behavioral Modeling and Simulation Workshop, pp. 13-18, 2009.

[32] I. Raafat, H. Ragaie, and D. Galayko, "Modeling of self-initialized electrostatic energy scavenger," IEEE, pp. 406-409, 2009.

[33] H. R. Florentino, R. C. S. Freire, and C. S. Florentino, "Optimization of control switch for energy harvest circuit using electrostatic charges," in Proc. 2010 IEEE Asia Pacific Conference Circuits and Systems, pp. 668-671, 2010.

[34] A. Kempitiya, D. A. Borca-Tasciuc, and M. M. Hella, "Analysis and optimization of asynchronously controlled electrostatic energy harvesters," Industrial Electronics, IEEE Transactions, vol. 59, no. 1, pp. 456-463, 2011.

[35] T. Sterken, K. Baert, G. Borghs, P. Fiorini, and R. Puers, "An electret-based electrostatic $\mu$-generator," in Proc. 12th International Conference on Solid State Sensors, Actuators and Microsystems, pp. 1291-1294, 2003.

[36] Y. Naruse, N. Matsubara, K. Mabuchi, M. Izumi, and K. Honma, "Electrostatic micro power generator from low frequency vibration such as human motion," Proceedings of Power MEMS, pp. 19-22, 2008.

[37] M. Edamoto, Y. Suzuki, N. Kasagii, K. Kashiwagi, Y. Morizawa, T. Yokoyama, T. Seki, and M. Oba, "Low-resonant-frequency micro electret generator for energy harvesting application," in Proc. Micro Electro Mechanical Systems IEEE 22nd International Conference, pp 1059-1062, 2009.

[38] D. Miki, Y. Suzuki, and N. Kasagi, "Effect of nonlinear external circuit on electrostatic damping force of micro electret generator," in Proc. Solid-State Sensors, Actuators and Microsystems Conference, pp 636-639, 2009.

[39] L. Bu, X. Wu, and L. Liu, "Collision based capacitive vibration energy harvesting," in Proc. Solid-State and Integrated Circuit Technology (ICSICT) 10th IEEE International Conference, pp. 1955-1957, 2010

[40] T. Suzuki, S. Nagasawa, H. Okamoto, and H. Kuwano, "Nove vibration-driven micro-electrostatic induction energy harvester With asymmetric multi-resonant spring," in Proc. IEEE Sensors 2010 Conference, pp. 1161-1164, 2010.

[41] G. Altena, M. Renaud, R. Elfrink, M. H. Goedbloed, C. D. Nooijer, and R. V. Schaijk, "Design improvements for an electret-based MEMS vibrational electrostatic energy harvester," Journal of Physics. Conference Series, vol. 476, no. 1, 2013.

[42] S. Meninger, J. M. Miranda, J. L. A. Chandrakasan, A. Slocum, M Schmidt, and R. Amirtharajah, "Vibration to electric energy conversion," IEEE Transactions Very Large Scale Integration (VLSI) Systems, vol. 9, pp. 64-76, 2001.

[43] J. O. M. Miranda, "Electrostatic vibration-to-electric energy conversion," PhD Thesis, Massachusetts institute of Technology, Massachusetts ,USA, 2004.

[44] P. D. Mitcheson, T. C. Green, and E. M. Yeatman, "Power processing circuits for electromagnetic, electrostatic and piezoelectric inertial energy scavengers," Microsyst Technol., pp. 1629-1635, 2004.

[45] S. P. Beeby, R. N. Torah, and M. J. Tudor, "Kinetic energy harvesting," in Proc. Act Workshop on Innovative Concepts. Esa-Estec, pp. 1-10, 2008.

[46] S. Roundy, P. K. Wright, S. Kristofer, and J. Pister, 'Micro-electrostatic vibration-to-electricity converters," ASME International Mechanical Engineering Congress, 2002.

[47] Y. M. Lim, B. Yang, R. K. Kotlanka, C. H. Heng, J. Xie, M. Tang, H. Han, H. Feng, and C. Lee, 'Theoretical study of the output energy for various MEMS based electrostatic mechanisms," Sustainable Energy Technologies, ICSET, pp. 552-557, 2008.

[48] C. P. Le and E. Halvorsen, "Electrostatic modeling of in-plane overlap energy harvesters," Power MEMS, Washington DC, USA, pp. 336-339, 2009.

[49] T. Tsutsumino, Y. Suzuki, and N. Kasagi, "Electromechanical modeling of micro electret generator for energy harvesting," in Proc. 
The 14th International Conference on Solid State Sensors, Actuator and Micro Systems, Lyon, France, pp. 863-866, 2007.

[50] H. Nguyen, D. Hah, P. R. Patterson, W. Piywattanametha, and M. C. Wu, "A novel MEMS tunable capacitor based on angular vertical comb drive actuators," Solid-State Sensor, Actuator and Microsystems Workshop, pp. 277-280, 2002.

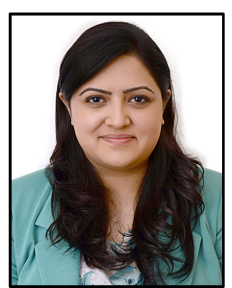

Rita Tareq Aljadiri was born in Diyala, Iraq, in 1984. She received the BEng degree (with honors) in electronics engineering from Coventry University/ Emirates Aviation College, Dubai, in 2008, and the MBA in aviation management from Coventry University in 2010.

In 2014, she received her $\mathrm{PhD}$ degree in electronic engineering from Coventry University, United Kingdom.

In 2005, Dr. Aljadiri joined the Engineering Department, Emirates Aviation College as electronic laboratory engineer, and in 2007 became an engineering lecturer. Her research interests include wind energy harvesting systems, electrostatic devices, power electronics, avionics systems, power processing and conditioning for low power systems.

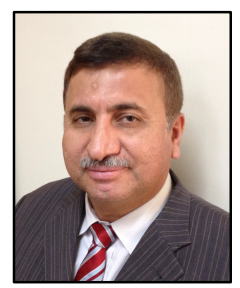

Luay Yassin Taha was born in Basra, Iraq, in 1959 $\mathrm{He}$ received the BSc degree in electrical engineering from the University of Basra, Iraq, in 1980 . He received the MSc degree in control engineering from the University of Technology Baghdad, Iraq, in 1985. In 2009 he received the Ph.D. degree in micro engineering and nanotechnology from the National university of Malaysia (UKM University), Bangi, Malaysia.

In 1986, he joined the scientific research council, centre of electronics and computer researches, as a researcher in homomorphic signal processing and in programmable instrumentation. In 1987 he joined the institute of technology, department of electronics and computer engineering as a Lecturer and in 1988 became a head of department. In September 1999 he became a senior lecturer at Dubai Aviation College, engineering department. In January 2002 he joined the Emirates Aviation College as a program manager in Electronics and Computer engineering department, and became an Assistance Professor in 2009.

Dr. Taha current research interests include electrostatic generators and piezoelectric micro generators. Dr. Taha is a Fellow of the Iraqi Engineering union and a member of the Institution of Electrical and Electronics Engineers (IEEE) and currently researcher in Windsor University, Ontario, Canada.

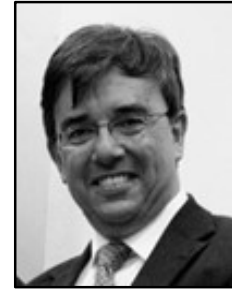

Paul Ivey is a Professor of Aerospace Engineering and currently he is Pro Vice-Chancellor Engagement, Enterprise and Research, Birmingham City University, Birmingham, United Kingdom.

Earlier he was the dean of faculty for Engineering and Computing $(\mathrm{E} \& \mathrm{C})$ at Coventry University. $\mathrm{He}$ has approximately 100 publications including 4 book contributions and 3 patents, has supervised over 20 $\mathrm{Ph} . \mathrm{D}$ studies and earned in excess of $£ 13 \mathrm{M}$ of research income. He is a visiting Professor to the Emirates Aviation College, Emirates Airline.

The $\mathrm{E} \& \mathrm{C}$ faculty has a turnover of $£ 40 \mathrm{M}$ and $3800 \mathrm{u} / \mathrm{g}, 580 \mathrm{p} / \mathrm{g}$, and 120 doctoral research students, another 5400 students educated overseas via 12 educational partnerships on 4 continents and comprises a High Performance Computing Facility, a Design Institute, the award winning SIGMA maths support centre (winner 2011 Times Higher Education Award for Outstanding Support for Students) and six departments: Civil Engineering Architecture and Building, Mechanical and Automotive Engineering, Computing, Engineering Management, Aerospace and Electrical Electronic Engineering and Mathematics and Control Engineering.

The faculty has worked with the Royal Academy of Engineering and MIT on two successive reviews of educational practice for Engineers and Technologists, and supports a Royal Academy of Engineering Academic Champion, 2 Royal Academy of Engineering Visiting Teaching Fellows and a Royal Academy of Engineering Visiting Professor. 